\title{
Fractal dimension analysis of grey matter in multiple sclerosis
}

\author{
Francisco J. Esteban ${ }^{\mathrm{a}, *}$, Jorge Sepulcre ${ }^{\mathrm{b}}$, Juan Ruiz de Miras ${ }^{\mathrm{c}}$, Juan Navas ${ }^{\mathrm{d}}$, Nieves Vélez de Mendizábal ${ }^{\mathrm{b}, \mathrm{e}}$, \\ Joaquín Goñi ${ }^{\text {b,f }}$, José $\mathrm{M}^{\mathrm{a}}$ Quesada $^{\mathrm{d}}$, Bartolome Bejarano ${ }^{\mathrm{b}}$, Pablo Villoslada ${ }^{\mathrm{b}, \mathrm{g}}$ \\ a Department of Experimental Biology, University of Jaén, Jaén, Spain \\ b Department of Neuroscience, CIMA, University of Navarra, Pamplona, Spain \\ c Department of Computer Science, University of Jaén, Jaén, Spain \\ d Department of Mathematics, University of Jaén, Jaén, Spain \\ e Department of Computational Sciences and Artificial Intelligence, University of the Basque Country, San Sebastian, Spain \\ ${ }^{\mathrm{f}}$ Department of Physics and Applied Mathematics, University of Navarra, Pamplona, Spain \\ ${ }^{g}$ Department of Neurology. Hospital Clinic, IDIBAPS, Barcelona, Spain
}

\section{A R T I C L E I N F O}

\section{Article history:}

Received 20 September 2008

Received in revised form 4 December 2008

Accepted 16 December 2008

Available online $\mathrm{xxxx}$

\section{Keywords:}

3D-fractal dimension

Box-counting method

Brain

Grey matter

Image analysis

Magnetic resonance imaging

Neurodegenerative disease

Multiple sclerosis

\begin{abstract}
A B S T R A C T
The fractal dimension (FD) is a quantitative parameter that characterizes the morphometric variability of a complex object. Among other applications, FD has been used to identify abnormalities of the human brain in conventional magnetic resonance imaging (MRI), including white matter abnormalities in patients with Multiple Sclerosis (MS). Extensive grey matter (GM) pathology has been recently identified in MS and it appears to be a key factor in long-term disability. The aim of the present work was to assess whether FD measurement of GM in T1 MRI sequences can identify GM abnormalities in patients with MS in the early phase of the disease. A voxel-based morphometry approach optimized for MS was used to obtain the segmented brain, where we later calculated the three-dimensional FD of the GM in MS patients and healthy controls. We found that patients with MS had a significant increase in the FD of the GM compared to controls. Such differences were present even in patients with short disease durations, including patients with first attacks of MS. In addition, the FD of the GM correlated with T1 and T2 lesion load, but not with GM atrophy or disability. The FD abnormalities of the GM here detected differed from the previously published FD of the white matter in MS, suggesting that different pathological processes were taking place in each structure. These results indicate that GM morphology is abnormal in patients with MS and that this alteration appears early in the course of the disease.
\end{abstract}

(c) 2008 Elsevier B.V. All rights reserved.

\section{Introduction}

Multiple Sclerosis (MS) is a chronic inflammatory and neurodegenerative disease of the central nervous system [1]. Although lesions involving the white matter (WM) are well recognized, recent studies have indicated extensive damage of the grey matter (GM) in MS, including microglia activation, cortical demyelination, and axonal, synaptic, and neuronal loss [2-7]. Recently, the extension and pathological basis of GM pathology have been highlighted from pathological and neuroimaging studies [8]. GM atrophy, extensively reported in MS, begins early and evolves over the course of the disease, and GM tissue volumes are lower in MS patients than in control subjects [9-15]. The study of GM damage is of critical importance since axonal and neuronal damage are the main factors responsible for long-term disability in MS.

\footnotetext{
* Corresponding author. Department of Experimental Biology; Systems Biology Unit; Faculty of Experimental and Health Sciences; University of Jaén; Campus Las Lagunillas s/n; 23071, Jaén, Spain. Tel.: +34 953212760; fax: +34 953211875.

E-mail address: festeban@ujaen.es (F.J. Esteban).
}

Neuroimaging studies have identified abnormalities in the GM of patients with MS, mainly volumetric changes. There are different approaches for detecting and quantifying the subtle neuropathological alterations taking place in the brain tissue in MS patients, including the magnetization-transfer ratio, diffusion-weighted imaging, or magnetic-resonance spectroscopy [16]. Recently, we found that fractal dimension (FD) identifies changes in the WM of MS patients, including the normal-appearing WM, even at the early stage of the disease [17]. FD is a measurement of the topological complexity of an object [18], and thus changes in the FD indicate alterations in the tissue structure under study. Because the WM has a highly complex anatomy such as the presence of axonal bundles, a pathological process that destroys brain tissue by creating an amorphous glial scar would decrease the FD of this tissue, as was the case of WM in MS [17]. Thus, FD can serve as a surrogate marker of the degree of brain damage.

The aim of the present study was to assess the FD of GM in healthy individuals and MS patients. Here, we focus primarily on patients at the early to intermediate phases of the disease in order to identify whether FD changes are an early phenomena in the disease course. For this goal, we developed a new computational algorithm 
for automatically detecting FD in 3D structures such as the brain, instead of using overlapping 2D images or creating a pseudo-3D analysis.

\section{Materials and methods}

\subsection{Patients}

A total of 52 patients with MS and 20 gender- and age-matched healthy controls were recruited by their neurologist (PV). All subjects gave their written informed consent according to the Helsinki Declaration before being included in the study, which was approved by the local Research Ethics Committee. Twenty patients suffered first attacks of MS (FAMS), fulfilling the criteria of dissemination in space and time [19]; and 32 had relapsing-remitting MS (RRMS). Patients with an active relapse, or who were taking corticosteroids or who had suffered a clinical relapse within the previous two months were excluded. MS-related disability was clinically assessed using the Extended Disability Status Scale (EDSS) [20] and the MS Functional Composite (MSFC) [21]. Demographic, clinical and MR image data of the subjects are shown in Table 1.

\subsection{MR acquisition}

In the following month of neurological assessment, a MR imaging study was performed on a 1.5-T SIEMENS SYMPHONY scanner (Erlangen, Germany). High-resolution volumetric images were acquired with a T1-weighted MR sequence (TR $2140 \mathrm{~ms}$, TE $5.04 \mathrm{~ms}$, flip angle $15^{\circ} ; 256 \times 256$ matrix size; 48 contiguous 3 -mm axial slices; 25 -cm FOV; in-plane resolution of $0.5 \times 0.5)$ and a proton density/T2weighted sequence (TR $3700 \mathrm{~ms}$, TE $29 \mathrm{~ms}$, turbo factor 5; 256×256 matrix size; 48 contiguous 3-mm axial slices; $25-\mathrm{cm}$ FOV; in-plane resolution of $0.5 \times 0.5$ ).

\subsection{Brain segmentation and volume of lesions}

A modified version of the optimized voxel-based morphometric (VBM) protocol [22] was used by a trained operator (JS) in order to obtain the normalized and segmented GM images from each subject's T1 MR, while avoiding the bias introduced by WM lesions in the normalization and segmentation procedures [23]. For this purpose we used SPM2 toolbox (Wellcome Department of Cognitive Neurology, University College of London, London, UK), www.fil.ion.ucl.ac.uk/spm running under Matlab v. 6.5 (Mathworks Inc., Natick, MA), and MRIcro software (Chris Rorden, University of Nottingham, Great Britain; http://www.sph.sc.edu/comd/rorden/mricro.html). In brief, a 3D binary MS lesion mask in native space was created at the voxel level for each patient (intra-class correlation coefficient $=0.892(p<0.001)$

Table 1

Baseline demographics of the MS patients and HC group

\begin{tabular}{|c|c|c|c|}
\hline & MS & $\mathrm{HC}$ & $p$ value \\
\hline $\bar{N}$ & 52 & 20 & \\
\hline $\mathrm{Age}^{\mathrm{a}}$ & $35.27 \pm 8.82$ & $37.35 \pm 8.66$ & ns \\
\hline Sex ratio, male:female & $16 / 36$ & $8 / 12$ & ns \\
\hline Disease duration ${ }^{\mathrm{b}}$, years & 2.20 ( 1 to 36$)$ & na & \\
\hline MS subtype & 20 FAMS/32 RRMS & na & \\
\hline EDSS $^{\mathrm{b}}$ & $2.0(0$ to 6.0$)$ & na & \\
\hline MSFC $^{\mathrm{a}}$ & $0.15 \pm 0.73$ & na & \\
\hline T1 lesion load ${ }^{\mathrm{b}}, \mathrm{cm}^{3}$ & $7.90(0.1$ to 96.41$)$ & na & \\
\hline T2 lesion load ${ }^{\mathrm{b}}, \mathrm{cm}^{3}$ & 36.84 ( 1.20 to 175.90$)$ & na & \\
\hline GM volume ${ }^{\mathrm{a}}, \mathrm{cm}^{3}$ & $522.67 \pm 52.33$ & $633.87 \pm 72.41$ & $<0.001$ \\
\hline
\end{tabular}

HC: Healthy control; MS: Multiple Sclerosis; CIS: Clinically Isolated Syndrome; RR: Relapsing-Remitting; EDSS: Expanded Disability Status; MSFC: MS Functional Composite; GM: grey matter. Data are expressed in a mean \pm standard deviation or ${ }^{\mathrm{b}}$ median (range) depending on the distribution of the variable. na: Not applicable. ns: Not statistically significant.
[17]. Later, we applied this lesion mask to regions containing visible lesions in every segmentation and normalization step in the VBM protocol [23]. In order to preserve the total within-voxel volume, all voxel signal intensities in the final GM segmented images were multiplied by the Jacobian determinants (Jacobian modulation) derived from the spatial normalization [22]. For each patient, we also measured the total volume of WM-lesion load on T1 and T2 studies using the MRIcro software. We calculated the total lesion volume in each patient by multiplying the total number of lesion voxels by the size of the voxel.

\subsection{D fractal dimension computation method}

The FD of the entire brain was determined from segmented GM in each subject. We selected the classical box-counting method [24] for computing the 3D-FD values because it can evaluate the FD of structures without self-similarity (the brain is not strictly self-similar; [25]). To achieve this, the corresponding 156 2D-MR images for each subject were stored in a $3 \mathrm{D}$ grid where each position $(X, Y, Z)$ is the value between 1 and 255 for the pixel $(X, Y)$ in the given image $Z$. These types of grids are typically referred to as 3D images or volumes [26]. For each box of size $N$, we construct 3D boxes by collecting $N+1$ consecutive pixels in the three dimensions of the grid. If all pixels located inside a box have a value greater than or equal to the specified threshold (see below), then the box is classified as BLACK; if the value of the pixels is lower than the specified threshold, then the box is classified as WHITE; otherwise, the box is classified as GREY (Fig. 1). Then, for each case of study and threshold, we classified boxes with sizes between 1 and 50, where the corresponding BLACK + GREY data constituted the basis for calculating the 3D-FD. The 3D-FD values were obtained through a $\log -\log$ linear regression in which the $\mathrm{X}$ axis represents the inverse of the size of the boxes and the $\mathrm{Y}$ axis represents the box counting for the type of box selected. The final value for the 3D-FD corresponds to the slope of this linear regression for the range of box sizes that maximizes the correlation value for the given threshold (Fig. 1). A previous manual segmentation of the images using ImageJ (http://rsbweb.nih.gov/ij/) showed 70 as the most appropriate threshold value (in a grey scale from 0 to 255); thus, the 3D-FD values finally obtained from each subject for the statistical analysis were those with 70 as the segmentation threshold, and from 6 to 16 as the range of box sizes (which maximizes the correlation for this threshold; Fig. 1).

The accuracy of our method for 3D-FD calculation was determined by evaluating three different 3D objects with well-known dimensions: a solid cube of size $320 \times 320 \times 320$ (exact topological dimension: 3 ), a solid sphere of diameter 320 (exact topological dimension: 3 ), and the Menger Sponge of size $320 \times 320 \times 320$ (exact fractal dimension: 2.7268 ). The $3 \mathrm{D}-\mathrm{FD}$ values resulting with our approach were 2.9876 , 2.9428 , and 2.725 , respectively; these results are very close to the theoretical values and similar to those detected using other published approaches to calculate the FD of cerebral structures [25,27].

The 3D grid generated from segmented 2D MRI slices, 3D box counting, 3D visualization and the 3D-FD computation and graphical representation has been implemented in $\mathrm{C}++$ language, and OpenGL library [28] was used for the graphical output. Other modules of our software performed the batch processes for computing the several 3D-FD for different box size ranges and thresholds over the collection of subjects. Finally, the results of the batch processes were stored in tab-delimited text files.

\subsection{Statistical analysis}

We used the Shapiro-Wilk test to determine the normal distribution of our variables and the Kruskal-Wallis and Mann-Whitney W test to compare the FD values between groups of subjects. Pearson's or Spearman's correlation tests (parametric and non-parametric, 
respectively) were applied to assess univariate correlation depending on the distribution of the variables. The level of significance for the results was set at $p=0.05$. These analyses were carried out using the statistical packages SPSS v13.0, or STATGRAPHICS Plus 5.1.

\section{Results}

We quantified the FD of the GM in a cohort of 52 patients with MS and 20 controls, using a new computational method for calculating the 3D-FD. Clinical demographics are shown in Table 1. The cohort was comprised of patients in the early to intermediate stages of the disease (FAMS: $n=20$; RRMS: $n=32$ ), except for one patient with long disease duration (36 years). In addition, patients had low to significant disability (median EDSS 2.0 [range: 0 to 6.0]). After calculating the 3DFD of the brain GM from each subject, we found that patients with MS had an increase in the FD of the GM compared to controls (MS FD: $2.67959 \pm 0.00103$; controls FD: $2.67146 \pm 0.00144 ; p<0.001$; Fig. 2 A). Such differences in the FD of the GM were found for either FAMS or RRMS compared to controls (FAMS FD: $2.676 \pm 0.0017$; RRMS FD:
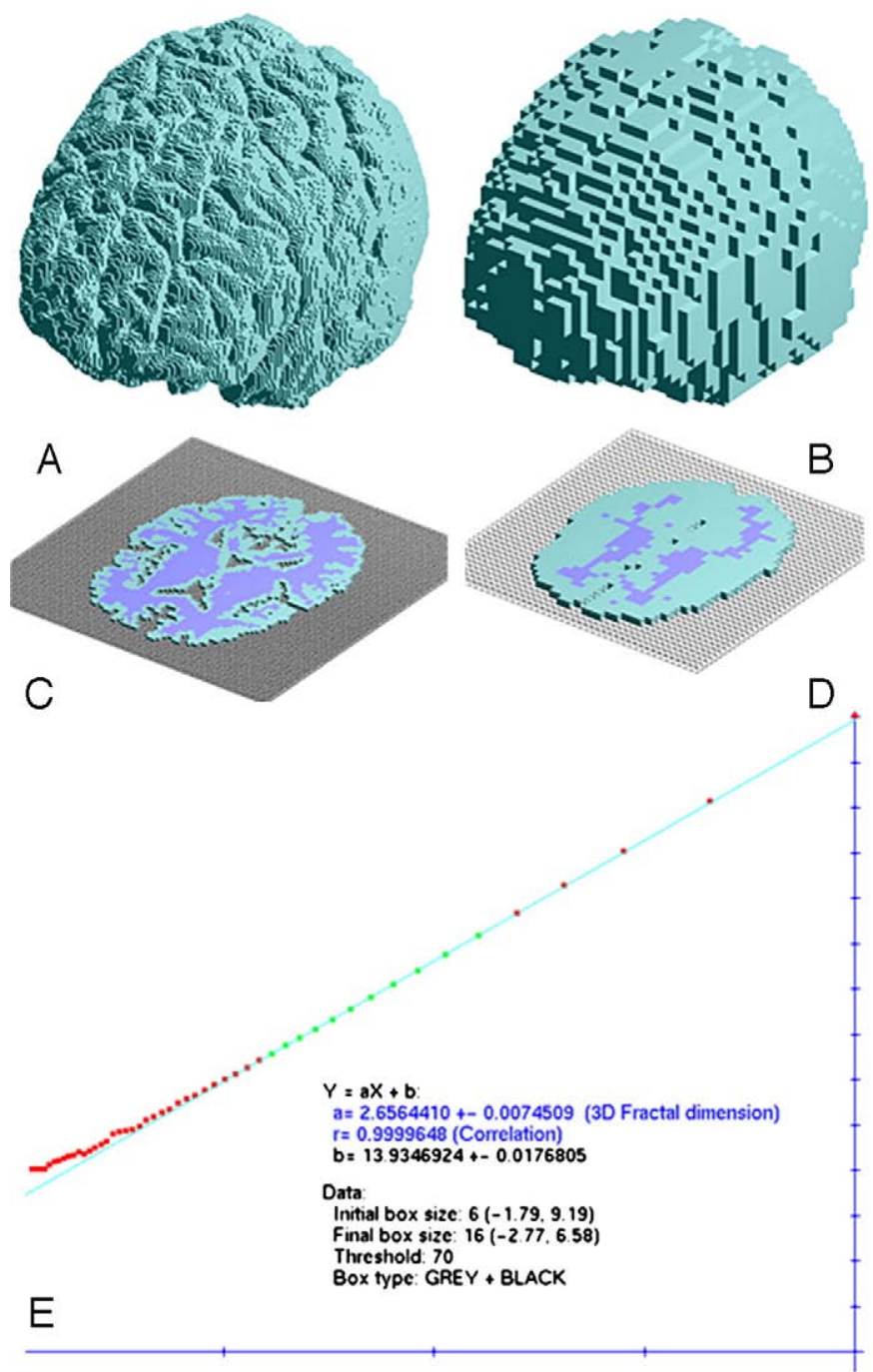

Fig. 1. Grid classification for a 3D image composed of $1562 \mathrm{D}$ slices of segmented grey matter with a threshold of 1 and for two different box sizes: 2 (A) and 5 (B); due to the 3D representation, all boxes shown are classified as GREY. Boxes of sizes 2 (C) and 5 (D) for only one row of the total classification, where the box classification in BLACK (blue colour), WHITE (empty colour) and GREY (green colour) is clearly shown. (E) Example of $3 \mathrm{D}$ fractal dimension computation through linear regression over the box counting; the range selected for the linear regression is the set of green points (box sizes from 6 to 16); red points are the box counting of box sizes not selected for the final computation.
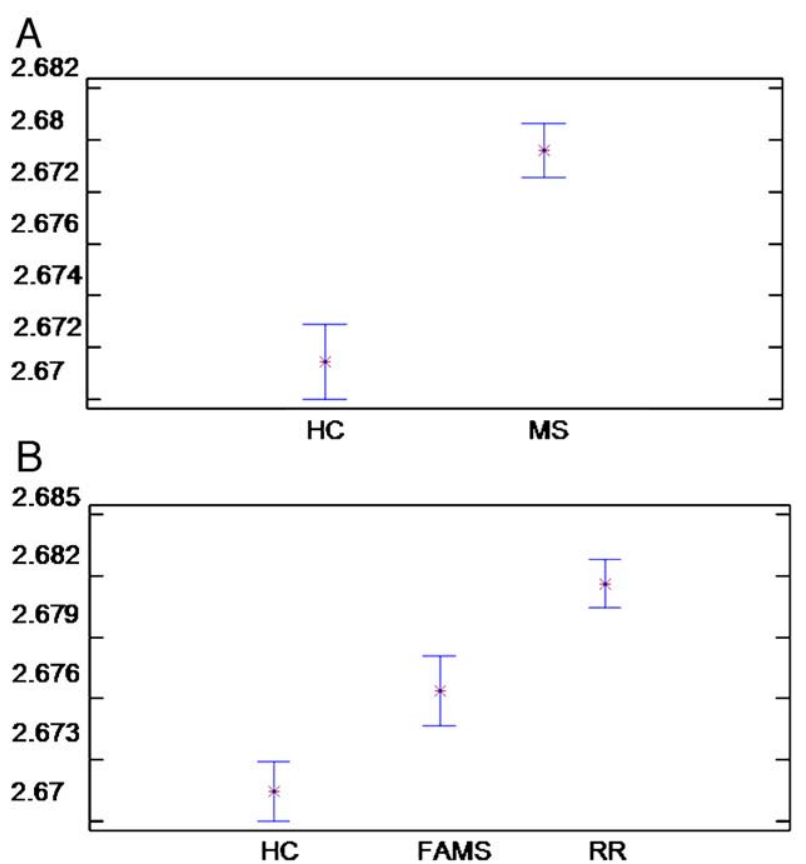

Fig. 2. Fractal dimension (FD) comparison of the grey matter (GM) corresponding to the full-range thresholded images from controls and patients. A) Patients with MS have higher FD of the cortical GM than healthy controls (HC). B) Comparison of the FD analysis between disease subtype and HC. Results are expressed as the mean (x) and SEM (bars) of the FD.

$2.681 \pm 0.0011)(p<0.05$ in both cases; Fig. 2B). Finally, we also found a significant difference in the GM FD between FAMS and RRMS patients $(p<0.05$; Fig. 2B).

Concerning the brain-lesion load, we found a statistically significant correlation between the FD of the GM and lesion volume, both on T1 $(r=0.435, p=0.005)$ and T2 $(r=0.329, p=0.017)$. On the other hand, the FD of the GM did not correlate with GM volume. Also, we investigated whether the changes in the FD would be associated with clinical variables. No correlation was detected between the FD of the grey matter and EDSS, MSFC scores, disease duration, or age (data not shown).

\section{Discussion}

In this study, we applied a new technique for analyzing GM pathology in MS, i.e. 3D-FD. Our results of higher FD values of the GM in MS patients compared to controls indicate that GM tissue in MS has higher morphological complexity, perhaps due to the presence of the inflammatory component (i.e. microglia activation) and cellular changes (synapse pruning, demyelination, brain-blood barrier changes, etc.) in the GM. This finding is striking because in our previous study assessing the FD of the WM, we found a decrease instead of an increase in the FD of the WM in MS patients [17]. This differential behavior in the FD between GM and WM in MS supports the concept that the damage mechanism or the response to tissue damage differs between GM and WM. Similar findings have been reported for other techniques studying GM in MS, such as GM atrophy, which was found to follow different dynamics between GM and WM in MS [15]. GM damage is of particular importance in MS, because tissue damage in GM is a large component of MS disease load and a key factor in long-term disability. Measurement of GM atrophy is a sensitive technique for identifying lesions in GM, as well as other nonconventional MRI techniques (MRS, DTI, MTR), when compared to the insensitivity of conventional MRI techniques (T1, T2, PD) for identifying GM damage $[15,29]$. Therefore, new methods able to quantify GM pathology would be very important for monitoring disease course. 
Based on our findings, FD studies represent a useful measurement to detect GM changes in the cerebral architecture, providing an overall measurement of anatomical changes due to MS, as previously shown at the WM level [17].

Abnormalities in GM in MS appear from the clinical onset of the disease and become more pronounced as the condition progresses [16]. Our study has been carried out including mainly patients with a short to medium course of the disease (FAMS and RRMS patients). Thus, our study indicates that FD of the GM can detect subtle changes already present in the early stages of the disease. The fact that differences increased from FAMS to RRMS also suggests that FD changes increase with disease evolution. However, we cannot predict the behavior of the FD of the GM in progressive patients because GM atrophy is highly prominent in this subgroup of patients and can influence the calculation of the FD. Future studies in patients with progressive disease together with longitudinal studies assessing the FD of the GM will clarify whether FD changes in fact evolve over the course of the disease or whether they behave differentially from other MRI abnormalities observed in MS, such as brain atrophy or lesion load.

An unexpected finding in our study was the lack of correlation between FD of the GM and GM atrophy, which might have several explanations. First, GM atrophy is less pronounced in the early stages of the disease and, because we studied patients with FAMS and RRMS, we might lack power to detect an association. Second, similarly to the fact that WM atrophy reduces the extension of WM tissue damage, GM atrophy might have also precluded the identification of GM tissue damage associated with FD changes.

Our study has some limitations. First, we analyzed patients with FAMS and RRMS in a cross-sectional study and therefore we cannot ascertain whether FD changes are more pronounced in progressive patients, or determine how such changes evolve with disease duration, or identify the effect of disease-modifying drugs in GM FD evolution. Secondly, we did not separately measure the FD of deep GM and cortical GM, which may be affected by different pathological processes. Third, GM is susceptible to the effect of aging and it is still not well known how age affects FD of the GM. This aspect is of special importance when studying patients with progressive forms of the disease or for prospective studies.

Taking into account that a GM pathology is common in MS $[5,15]$, the FD increased in MS detected here could be related to an increase in GM abnormalities, as has been also reported in association with the irregularities of the GM structure in a FD analysis recently carried out in schizophrenia [30]. In addition, GM histological abnormalities increase in MS over time [8], which might account for the significant increase of the FD in the transition from FAMS to RRMS. The topography of GM damage in MS drives the appearance of functional deficits [31,32]. We speculate that this might explain why in our study an overall measurement of GM damage such as FD does not correlate with disability, which depends on the topography of the brain damage (i.e. corticospinal pathway for motor function). Localized measurement of FD might provide complimentary information or identifying more selectively damaged cortical regions, since cortical atrophy does not appear to be a uniform process throughout the GM [23,33-35].

Cortical damage appears to evolve independently of WM damage in MS [15,36] and our study showing a different behavior of the FD between GM and WM also supports this concept. Thus, an unanswered question is whether GM atrophy occurs as a direct result of GM pathology or whether it is secondary to tissue damage within WM lesions. The fact that the FD of the GM correlates with WM lesion load, especially in T1 sequences that are strongly associated with axonal damage, suggests that at least some of the changes in its architecture is secondary to axonal damage in the WM. This result agrees with our previous finding that axonal damage in the visual radiations explains up to one third of GM atrophy in some particular regions [37]. However, it can also be argued that this correlation is due to disease activity, meaning that patients with more active disease have more lesion load in the WM and more GM abnormalities. On the other hand, the FD of the GM did not correlate with GM volume, even though we have detected a decrease of the GM volume in MS; this GM volume decrease is higher than previously reported $[10,11]$, but this variation in range may be related to the different processing of the data.

In conclusion, a better understanding of the biological basis of GM damage is essential in order to develop new therapies aimed to prevent disability accumulation. New neuroimaging and pathological studies are providing fresh insights into the pathogenesis of GM damage in MS.

\section{Acknowledgements}

We would like to thank Diego Maza, from the Department of Physics of the University of Navarra for his helpful comments. This work was supported by Junta de Andalucía (BIO-302) to FJE, the Spanish Ministry of Health (FIS RD07/0060/0001) and Fundacion Uriach to PV, and partially granted by the Spanish Ministry of Science and Technology and the European Union by means of ERDF funds (TIN200406326-C03-03; TIN2007-67474-C03-03) to JRdM. JS was a fellow of the Spanish Ministry of Health (FIS CM\#05/00222), NVM of the Basque Country Government and JG of the Navarra Government. Authors have no conflicting financial interests.

\section{References}

[1] Steinman L. Multiple sclerosis: a two-stage disease. Nat Immunol 2001;2:762-4.

[2] Kidd D, Barkhof F, McConnel R, Algra PR, Allen IV, Revesz T. Cortical lesions in multiple sclerosis. Brain 1999;122:17-26.

[3] Peterson JW, Bö L, Mörk S, Chang A, Trapp BD. Transected neuritis, apoptotic neurons, and reduced inflammation in cortical multiple sclerosis lesions. Ann Neurol 2001;50:389-400.

[4] Kutzelnigg A, Lucchinetti CF, Stadelmann C, Brück W, Rauschka H, Bergmann M, et al. Cortical demyelination and diffuse white matter injury in multiple sclerosis. Brain 2005;128:2705-12.

[5] Wegner C, Esiri MM, Chance SA, Palace J, Matthews PM. Neocortical neuronal, synaptic, and glial loss in multiple sclerosis. Neurology 2006;67:960-7.

[6] Pirko I, Lucchinetti CF, Sriram S, Bakshi R. Gray matter involvement in multiple sclerosis. Neurology 2007;68:634-42.

[7] Stadelmann C, Brück W. Interplay between mechanisms of damage and repair in multiple sclerosis. J Neurol 2008;255:12-8.

[8] Geurts JJ, Barkhof F. Grey matter pathology in multiple sclerosis. Lancet Neurol 2008;7:841-51.

[9] Ge Y, Grossman RI, Udupa JK, Babb JS, Nyúl LG, Kolson DL. Brain atrophy in relapsing-remitting multiple sclerosis: fractional volumetric analysis of gray matter and white matter. Radiology 2001;220:606-10.

[10] Chard DT, Griffin CM, Parker GJ, Kapoor R, Thompson AJ, Miller DH. Brain atrophy in clinically early relapsing-remitting multiple sclerosis. Brain 2002;125:327-37.

[11] Chard DT, Griffin CM, Rashid W, Davies GR, Altmann DR, Kapoor R, et al. Progressive grey matter atrophy in clinically early relapsing-remitting multiple sclerosis. Mult Scler 2004;10:387-91.

[12] Dalton CM, Chard DT, Davies GR, Miszkiel KA, Altmann DR, Fernando K, et al. Early development of multiple sclerosis is associated with progressive grey matter atrophy in patients presenting with clinically isolated syndromes. Brain 2004; 127:1101-7.

[13] Tedeschi G, Lavorgna L, Russo P, Prinster A, Dinacci D, Savettieri G, et al. Brain atrophy and lesion load in a large population of patients with multiple sclerosis. Neurology 2005;65:280-5.

[14] Tiberio M, Chard DT, Altmann DR, Davies G, Griffin CM, Rashid W, et al. Gray and white matter volume changes in early RRMS: a 2-year longitudinal study. Neurology 2005;64:1001-7.

[15] Fisher E, Lee JC, Nakamura K, Rudick RA. Gray matter atrophy in multiple sclerosis: a longitudinal study. Ann Neurol 2008;64:255-65.

[16] Miller DH, Thompson AJ, Filippi M. Magnetic resonance studies of abnormalities in the normal appearing white matter and grey matter in multiple sclerosis. J Neurol 2003;250:1407-19.

[17] Esteban FJ, Sepulcre J, de Mendizábal NV, Goñi J, Navas J, de Miras JR, et al. Fractal dimension and white matter changes in multiple sclerosis. Neuroimage 2007;36:543-9.

[18] Fernández E, Jelinek HF. Use of fractal theory in neuroscience: methods, advantages, and potential problems. Methods 2001;24:309-21.

[19] Polman CH, Reingold SC, Edan G, Filippi M, Hartung HP, Kappos L, et al. Diagnostic criteria for multiple sclerosis: 2005 revisions to the "McDonald Criteria". Ann Neurol 2005;58:840-6.

[20] Kurtzke JF. Rating neurologic impairment in multiple sclerosis: an expanded disability status scale (EDSS). Neurology 1983;33:1444-52.

[21] Fischer JS, Rudick RA, Cutter GR, Reingold SC. The Multiple Sclerosis Functional Composite Measure (MSFC): an integrated approach to MS clinical outcome 
assessment. National MS Society Clinical Outcomes Assessment Task Force. Mult Scler 1999;5:244-50.

[22] Good CD, Johnsrude IS, Ashburner J, Henson RN, Friston KJ, Frackowiak RS. A voxelbased morphometric study of ageing in 465 normal adult human brains. Neuroimage 2001;14:21-36.

[23] Sepulcre J, Sastre-Garriga J, Cercignani M, Ingle GT, Miller DH, Thompson AJ. Regional gray matter atrophy in early primary progressive multiple sclerosis: a voxel-based morphometry study. Arch Neurol 2006;63:1175-80.

[24] Hou X, Gilmore R, Mindlin GB, Solari HG. An efficient algorithm for fast $O\left(N^{*} \ln (N)\right)$ box counting. Phys-Lett-A 1990;151:43-6.

[25] Zhang L, Liu JZ, Dean D, et al. A three-dimensional fractal analysis method for quantifying white matter structure in human brain. J Neurosci Methods 2006;150:242-53.

[26] Chen M, Kaufman AE, Yagel R. Volume graphics. New York: Springer; 2000.

[27] Jiang J, Zhu W, Shi F, Zhang Y, Lin L, Jiang T. A robust and accurate algorithm for estimating the complexity of the cortical surface. J Neurosci Methods 2008;172:122-30.

[28] Shreiner D. OpenGL reference manual: the official reference document to OpenGL, version 1.4. 4th ed. Addison-Wesley Professional; 2004.

[29] Geurts JJ, Bo L, Pouwels PJ, Castelijns JA, Polman CH, Barkhof F. Cortical lesions in multiple sclerosis: combined postmortem MR imaging and histopathology. AJNR Am J Neuroradiol 2005;26:572-7.

[30] Sandu AL, Rasmussen Jr IA, Lundervold A, Kreuder F, Neckelmann G, Hugdahl K, et al. Fractal dimension analysis of MR images reveals grey matter structure irregularities in schizophrenia. Comput Med Imaging Graph 2008;32:150-8.
[31] Amato MP, Bartolozzi ML, Zipoli V, Portaccio E, Mortilla M, Guidi L, et al. Neocortical volume decrease in relapsing-remitting MS patients with mild cognitive impairment. Neurology 2004;63:89-93.

[32] Benedict RH, Bruce JM, Dwyer MG, Abdelrahman N, Hussein S, Weinstock-Guttman $\mathrm{B}$, et al. Neocortical atrophy, third ventricular width, and cognitive dysfunction in multiple sclerosis. Arch Neurol 2006;63:1301-6.

[33] Prinster A, Quarantelli M, Orefice G, Lanzillo R, Brunetti A, Mollica C, et al. Grey matter loss in relapsing-remitting multiple sclerosis: a voxel-based morphometry study. Neuroimage 2006;29:859-67.

[34] Tekok-Kilic A, Benedict RH, Weinstock-Guttman B, Dwyer MG, Carone D, Srinivasaraghavan B, et al. Independent contributions of cortical gray matter atrophy and ventricle enlargement for predicting neuropsychological impairment in multiple sclerosis. Neuroimage 2007;36:1294-300.

[35] Mesaros S, Rovaris M, Pagani E, Pulizzi A, Caputo D, Ghezzi A, et al. A magnetic resonance imaging voxel-based morphometry study of regional gray matter atrophy in patients with benign multiple sclerosis. Arch Neurol 2008;65:1223-30.

[36] Bö L, Geurts JJ, van der Valk P, Polman C, Barkhof F. Lack of correlation between cortical demyelination and white matter pathologic changes in multiple sclerosis. Arch Neurol 2007;64:76-80.

[37] Sepulcre J, Goñi J, Masdeu J, Bejarano B, Velez N, Toledo J, Villoslada P. Contribution of white matter lesions to grey matter atrophy in multiple sclerosis: evidence from voxel-based analysis of T1 lesions in the visual pathway. Arch Neurol; in press. 